\title{
Angiotensin receptor-neprilysin inhibitor for the treatment of heart failure: a review of recent evidence
}

\author{
Hong-Mi Choi ${ }^{1}$ and Mi-Seung Shin ${ }^{2}$
}

${ }^{1}$ Division of Cardiology, Hallym University Sacred Heart Hospital, Hallym University College of Medicine, Anyang; ${ }^{2}$ Division of Cardiology, Department of Internal Medicine, Gil Medical Center, Gachon University College of Medicine, Incheon, Korea

\section{Received: March 21, 2020}

Accepted: April 22, 2020

\section{Correspondence to}

Mi-Seung Shin, M.D.

Division of Cardiology, Department of Internal Medicine, Gil Medical Center, Gachon University College of Medicine, 21 Namdong-daero 774beon-gil, Namdong-gu, Incheon 21565, Korea

Tel: $+82-32-460-3663$

Fax: +82-32-469-1906

E-mail: msshin@gilhospital.com

https://orcid.org/0000-0002-

0273-0109

This paper was contributed by The Korean Society of Heart Failure.
Heart failure $(\mathrm{HF})$ is a growing health concern in aging societies worldwide. Sacubitril/valsartan is changing the real-world treatment in the whole spectrum of HF. The beginning was the PARADIGM-HF trial published in 2014, which demonstrated the beneficial effects of inhibiting natriuretic peptide breakdown in combination with hindering the renin-angiotensin system in HF patients with a reduced ejection fraction. Subsequent large-scale randomized trials have evaluated angiotensin receptor-neprilysin inhibitor in HF patients with acute decompensation or with preserved ejection fraction. The post hoc analyses are being conducted as well. This review summarizes the recent evidence of sacubitril/ valsartan regarding patient-centered outcomes, based on randomized controlled trials and their associated studies.

Keywords: Heart failure; Angiotensin receptor inhibitor; Neprilysin

\section{INTRODUCTION}

Heart failure (HF) is the primary cause of cardiovascular mortality worldwide. The prevalence of HF is approximately $1.5 \%$ in Korea, with similar rates in other developed countries $[1,2]$. However, the incidence of HF has steadily increased over the decades, especially among aging populations. Although significant advancements in the treatment of HF have been achieved, the HF-related mortality has seen little improvement [3].

In 2014, the first large scale randomized controlled trial (RCT) of LCZ696 was published [4]. The Prospective
Comparison of angiotensin receptor-neprilysin inhigitor (ARNI) with angiotensin converting enzyme inhibitor (ACEI) to Determine Impact on Global Mortality and Morbidity in Heart Failure (PARADIGM-HF) trial showed that ARNI could change the grim prognosis of patient with HF. These findings led to changes in the treatment guidelines for HF in Europe and the United States in 2016 and 2017, respectively, which recommended replacing ACEIs and angiotensin receptor blockers (ARBs) with ARNI for ambulatory HF with reduced ejection fraction (HFrEF) [5,6]. Follow-up studies are underway to assess extended indications for ARNI as well as to investigate its 
mechanism of beneficial action. In accordance with these results, the use of sacubitril/valsartan is rapidly increasing worldwide [7]. In this review, we summarize the recent evidence on the effects of ARNI on patient-centered outcomes from RCTs and associated studies.

\section{MECHANISM OF THE EFFECT OF ANGIOTENSIN RECEPTOR-NEPRILYSIN INHIBITOR ON THE PATHOPHYSIOLOGY OF HEART FAILURE}

\section{Neurohormonal activation in the pathophysiology of heart failure}

Traditionally, the pathophysiology of HF was largely understood from a hemodynamic perspective. Peripheral vasoconstriction as well as sodium and water retention were thought to contribute to the maintenance of intravascular volume in response to pump failure. Accordingly, patients with HF were mainly treated with inotropes, vasodilators and diuretics. However, the hemodynamic model did not fully explain the progressive deterioration in HF. Moreover, the medications for ameliorating hemodynamic change have not improved the prognosis of HF patients [8,9]. As additional evidence has emerged, the concept of HF as a neurohormonal disorder was proposed by Packer [10] in 1992. The sympathetic nervous system (SNS) and renin-angiotensin-aldosterone system (RAAS) were identified as the first and second neurohormonal axes, respectively, that are activated to compensate for pump failure in HFrEF. Although this mechanism helps the body maintain cardiac output in the short term, sustained activation of the SNS and RAAS can have adverse effects on the heart and other organs. This concept was supported by the findings that RAAS blockers such as ACEI, ARB, and aldosterone antagonist, as well as beta-blockers improved the survival of HFrEF patients [8].

The third axis of the neurohormonal mechanism is the natriuretic peptide (NP) system, which acts in opposition to RAAS and SNS activation. NPs exert protective effects on the heart and vasculature with increasing the level of urinary cyclic guanosine monophosphate, which not only enhances natriuresis and vasodilation but also reduces SNS and RAAS activities and inhibits adverse cardiac remodeling (Fig. 1) [11]. The effect of the
NP system enhancement was evaluated in patients with acute HF by the administration of nesiritide, a recombinant human B-type NP (BNP). However, the Acute Study of Clinical Effectiveness of Nesiritide in Decompensated Heart Failure (ASCEND-HF) found that nesiritide did not decrease mortality or rehospitalization rates and resulted in an increased risk of hypotension, leading to its discontinuation in 2018 [12]. An alternate therapeutic target for NP system activation is inhibition of neprilysin, which mediates the breakdown of NPs.

\section{The development of angiotensin receptor-neprilysin inihibitor}

ARNI is a combination drug, which consists of a neprilysin inhibitor and an ARB. The first-in-class ARNI sacubitril/valsartan (formerly known as LCZ696) is composed of sacubitril, the prodrug to sacubitrilat (neprilysin inhibitor), and valsartan (ARB). Neprilysin is a versatile enzyme that cleaves vasodilating peptides such as NPs and bradykinin, as well as vasoconstricting peptides such as angiotensin I and II [13]. Therefore, studies have revealed that inhibition of neprilysin alone failed to improve the prognosis of HF, likely due to its counterbalance effect [14,15]. Since inhibition of angiotensin I and II is a strategy to improve the survival of HFrEF patients, the need for simulataneoous inhibition of RAAS and NP system emerged.

Omapatrilat is a combination drug comprising an ACEI and neprilysin inhibitor. The Omapatrilat Versus Enalapril Randomized Trial of Utility in Reducing Events (OVERTURE) study compared the efficacies of omapatrilat and enalapril in 5,770 patients with class II-IV HFrEF according to the New York Heart Association (NYHA) classification system. The OVERTURE trial assessed the primary composite endpoint of death from any cause or HF hospitalization requiring intravenous treatment. Omapatrilat reduced the risk of death or hospitalization for cardiovascular causes compared with enalapril but failed to show improved efficacy over enalapril in terms of the primary endpoint (hazard ratio [HR], 0.94; 95\% confidence interval [CI], 0.86 to $1.03 ; p=0.187)[16]$.

Moreover, the Omapatrilat Cardiovascular Treatment Versus Enalapril (OCTAVE) trial, which evaluated the efficacies of omapatrilat and enalapril in patients with untreated or uncontrolled hypertension, showed that omapatrilat had better efficacy as an antihypertensive medication; however, patients treated with omapatrilat 


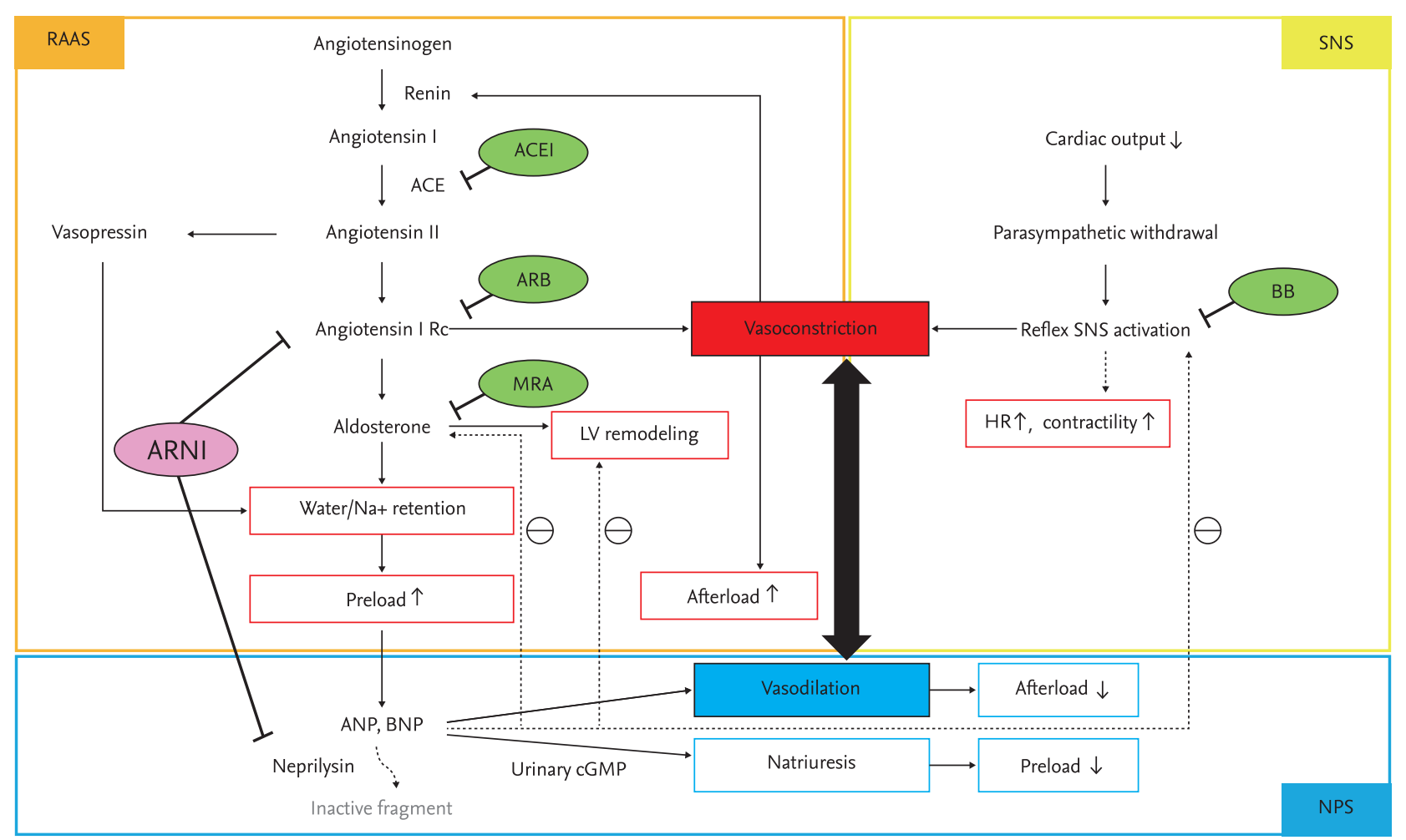

Figure 1. Three axes of the neurohormonal mechanism in heart failure. RAAS, renin-angiotensin-aldosterone system; ACEI, angiotensin converting enzyme inhibitor; ARB, angiotensin receptor blocker; Rc, receptor; MRA, mineralocorticoid receptor antagonist; LV, left ventricular; ARNI, angiotensin receptor-neprilysin inhibitor; ANP, atrial natriuretic peptide; BNP, brain natriuretic peptide; cGMP, cyclic guanosine monophosphate; SNS, sympathetic nervous system; BB, beta-blocker; HR, heart rate; NPS, natriuretic peptide system.

were three times more likely to experience angioedema compared with enalapril [17]. Since both neprilysin and angiotensin-converting enzyme metabolize bradykinin, the increased level of bradykinin, which is associated with inhibiting multiple degradation pathways, may result in more frequent and severe angioedema [18]. Given this adverse effect, an alternative to ACEIs that inhibit downstream RAAS by blocking angiotensin II type I receptors was established, creating the new ARNI drug class.

\section{RESULTS FROM RANDOMIZED CONTROLLED TRIALS OF ANGIOTENSIN RECEPTOR- NEPRILYSIN INHIBITOR IN PATIENTS WITH HEART FAILURE}

\section{Heart failure with reduced ejection fraction}

The PARADIGM-HF trial was a double-blind RCT that compared the long-term efficacy and safety of sacubi- tril/valsartan with those of enalapril in 8,399 patients with HFrEF. The study included NYHA class II-IV patients with a left ventricular ejection fraction (LVEF) $\leq 40 \%$ and increased BNP or N-terminal pro-B-type NP (NT-proBNP) level [19]. The PARADIGM-HF trial was prematurely terminated at a median follow-up of 27 months, because the benefit of sacubitril/valsartan exceeded the prespecified endpoint at the interim analysis (Table 1 and Fig. 2) [4]. The primary composite endpoint was cardiovascular death or hospitalization for $\mathrm{HF}$, and the study found that sacubitril/valsartan treatment resulted in an $\mathrm{HR}$ of $0.8 \mathrm{O}$ (95\% CI, 0.73 to 0.87 ; $p<0.001$ ) compared with enalapril for the primary endpoint. The HRs for all-cause death and cardiovascular death were 0.84 (95\% CI, 0.76 to $0.93 ; p<0.001$ ) and 0.80 (95\% CI, 0.71 to $0.89 ; p<0.001$ ), respectively. The effects of sacubitril/valsartan on the primary endpoint were consistent among nearly all prespecified subgroups. Hypotension developed more often in patients treat- 


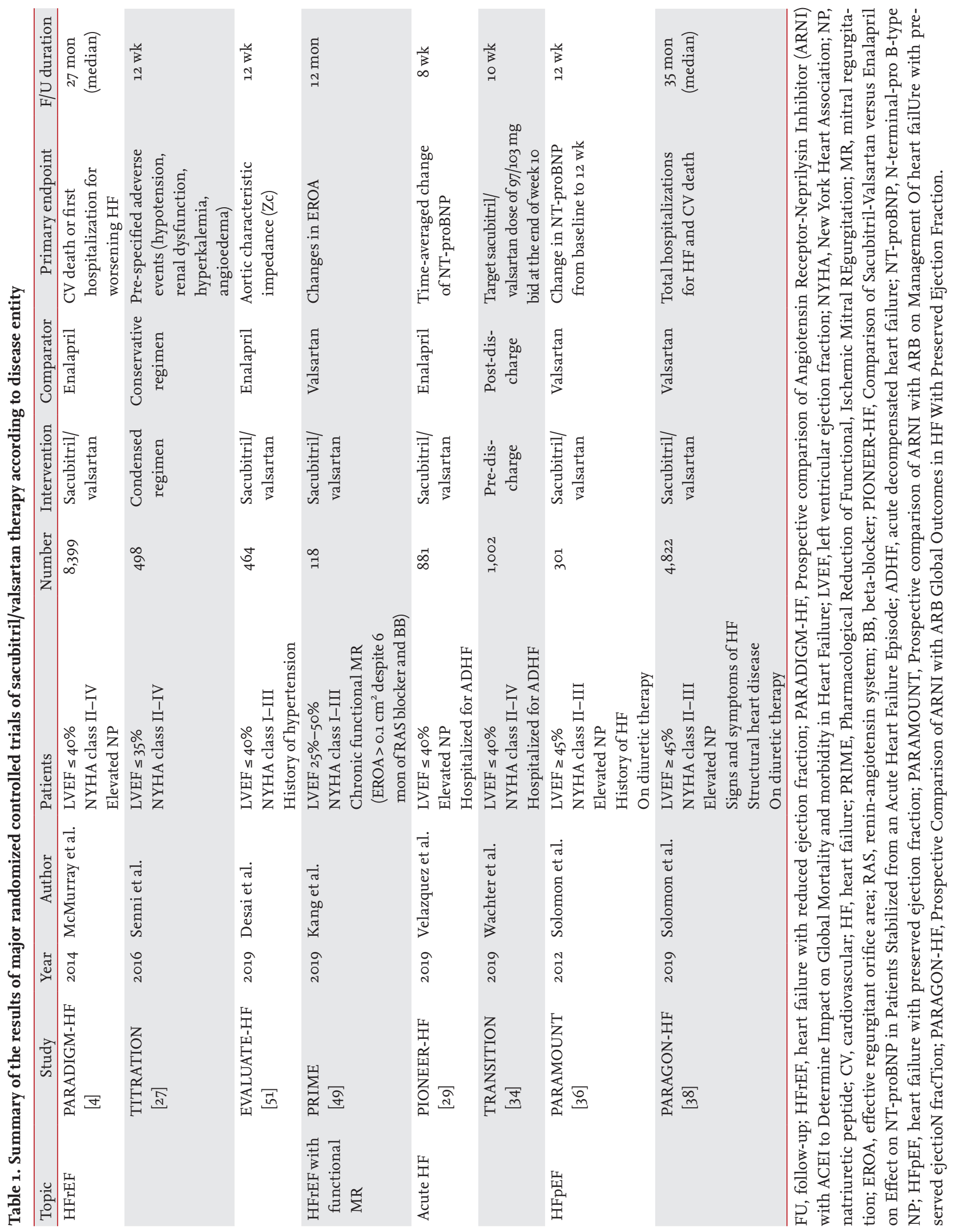




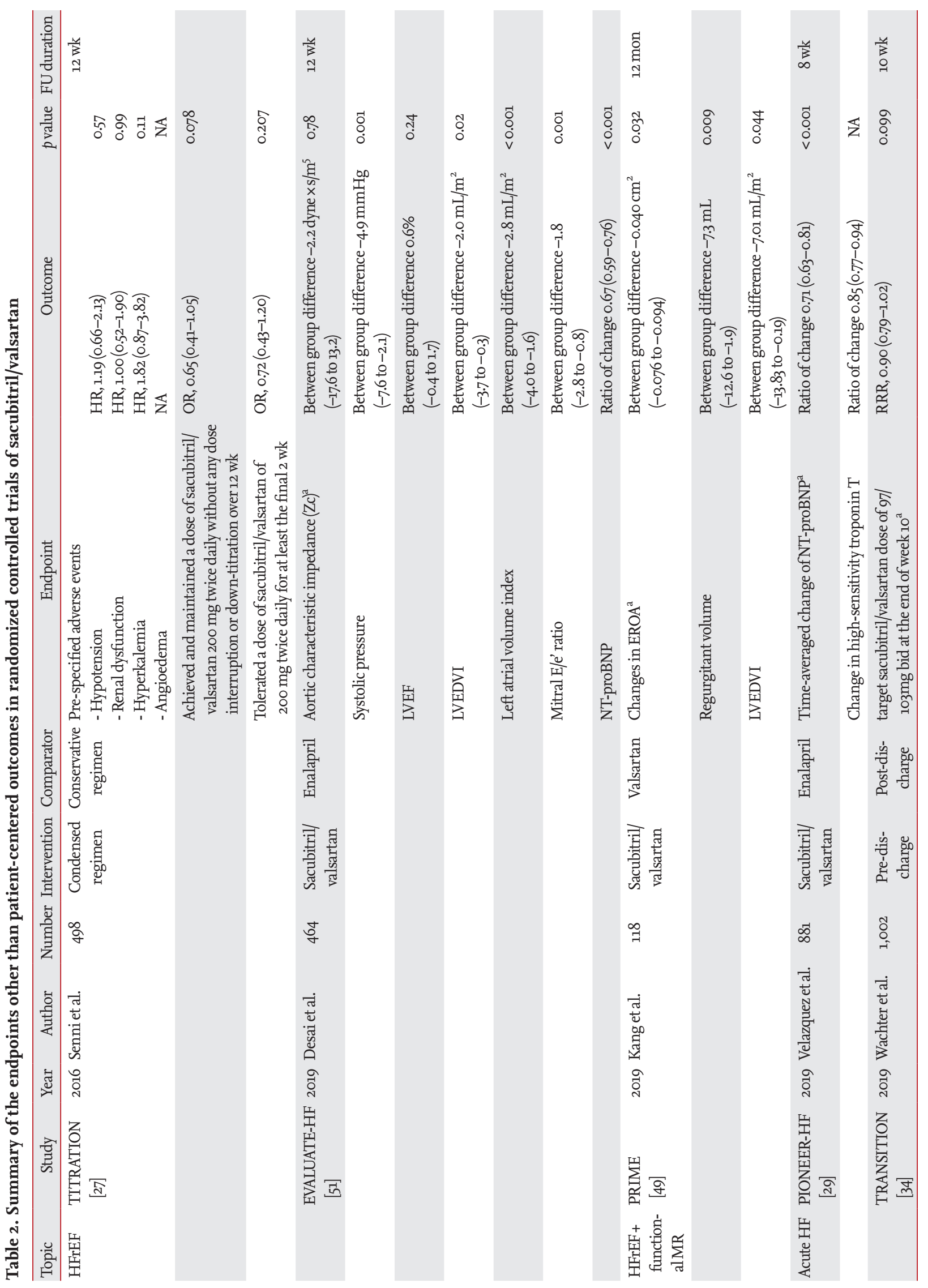




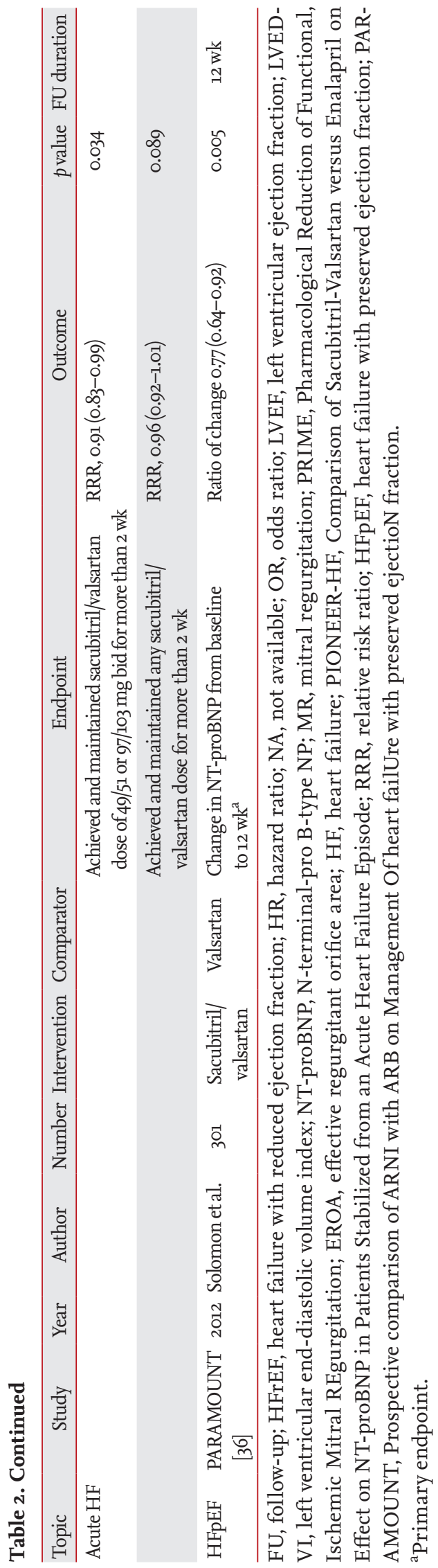

ed with sacubitril/valsartan, but modest increases in the serum creatinine level ( $\geq 2.5 \mathrm{mg} / \mathrm{dL}$ ) occurred less frequently in the sacubitril/valsartan group compared with the enalapril group. These findings were significant, as the comparator, enalapril, had been shown to reduce the mortality and hospitalization of HF patients with an LVEF $\leq 35 \%$ in the Studies of Left Ventricular Dysfunction (SOLVD) trial [20]. In addition, patients enrolled in the PARADIGM-HF trial received ACEI/ ARB, beta-blockers, and mineralocorticoid receptor antagonists when clinically indicated. These findings suggested that inhibiting neprilysin as well as the RAAS and SNS reduced the risks of death and hospitalization among patients with HFrEF.

In the PARADIGM-HF trial post hoc analysis, sacubitril/valsartan notably reduced the risks of both sudden cardiac death (HR, o.80; 95\% CI, 0.68 to $0.94 ; p=0.008$ ) and death from worsening $\mathrm{HF}$ (HR, 0.79; 95\% CI, o.64 to $0.98 ; p=0.034$ ) compared with enalapril. In contrast, there were no significant differences in the incidence of non-cardiovascular death or cardiovascular death from myocardial infarction or stroke between the sacubitril/ valsartan and enalapril groups [21]. While the underlying mechanism of sacubitril/valsartan remains unclear, the activated NP system promotes left ventricular reverse remodeling and reduced myocardial fibrosis, which may prevent the development of fatal arrhythmias [22]. Moreover, sacubitril/valsartan reduced the inducibility of ventricular arrhythmia and restored the expression of downregulated potassium channels in an experimental model of myocardial infarction in rats [23]. These results identify possible mechanisms associated with the reduced sudden cardiac death observed in the sacubitril/valsartan treatment groups.

The PARADIGM-HF trial post hoc analysis by Packer et al. [24] showed that sacubitril/valsartan reduced the risk of nonfatal clinical deterioration in surviving patients with HF. Fewer patients treated with sacubitril/valsartan required escalation of medical therapy for HF compared with those treated with enalapril. The sacubitril/valsartan group experienced lower rates of emergency department visits for $\mathrm{HF}$, intensive care, intravenous inotropes and cardiovascular or all-cause hospitalization compared with the enalapril group [24]. Furthermore, the time to first hospitalization for HF was significantly reduced within the first 30 days after randomization (HR, o.6o; 


\section{KJIM'}

The Korean Journal of Internal Medicine. Vol. 35, No. 3, May 2020

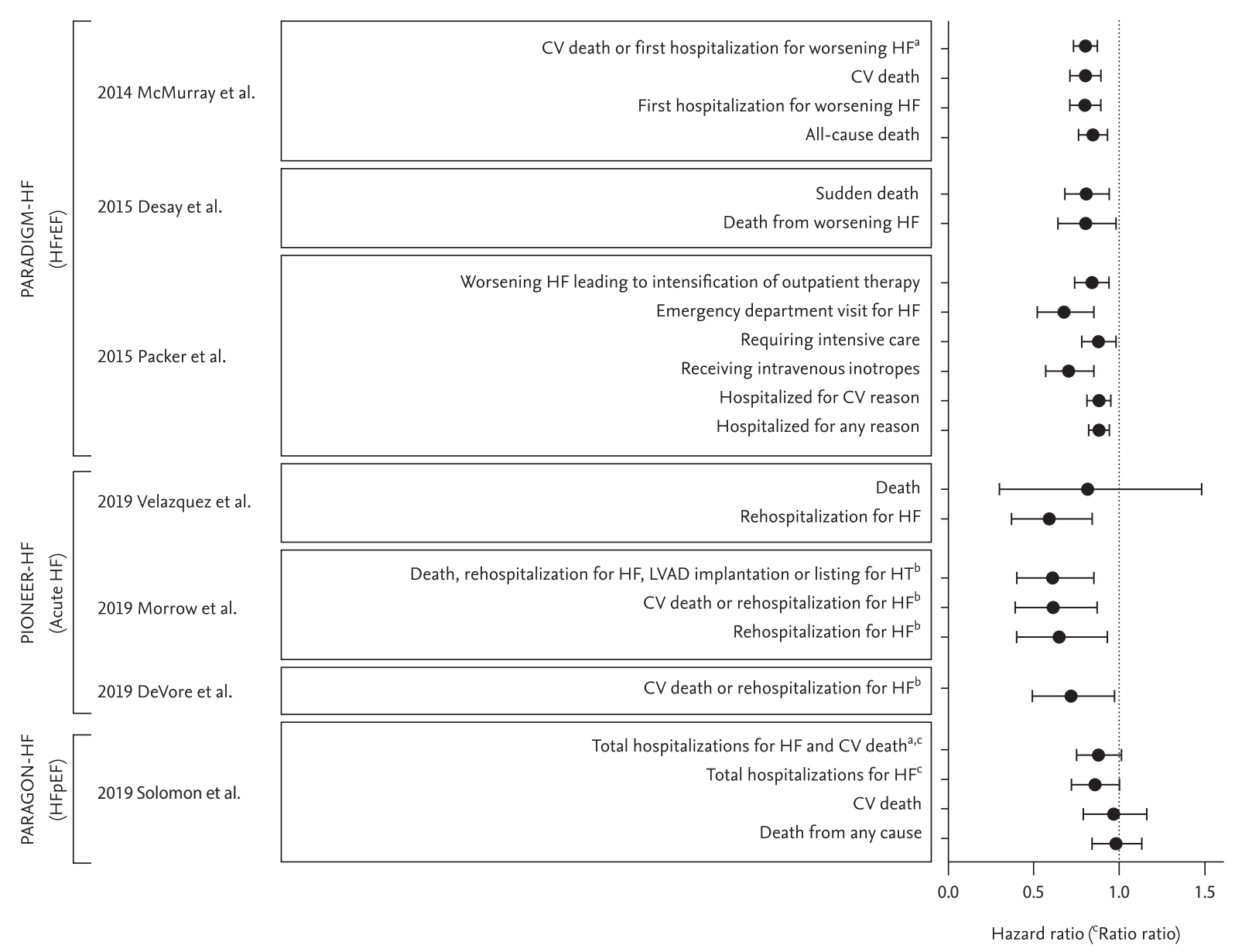

Figure 2. Patient-centered outcomes in major randomized controlled trials and their sub-analyses of sacubitril/valsartan. CV, cardiovascular; HF, heart failure; PARADIGM-HF, Prospective comparison of Angiotensin Receptor-Neprilysin Inhibitor (ARNI) with ACEI to Determine Impact on Global Mortality and morbidity in Heart Failure; HFrEF, heart failure with reduced ejection fraction; PIONEER-HF, Comparison of Sacubitril-Valsartan versus Enalapril on Effect on NT-proBNP in Patients Stabilized from an Acute Heart Failure Episode; LVAD, left ventricular assist device; HT, heart transplantation; PARAGON-HF, Prospective Comparison of ARNI with ARB Global Outcomes in HF With Preserved Ejection Fraction; HFpEF, heart failure with preserved ejection fraction. ${ }^{\text {a Primary endpoint. }}{ }^{\mathrm{b}}$ Adjudicated outcome by a blinded clinical event committee. ${ }^{\mathrm{c}}$ Rate ratio.

95\% CI, 0.38 to 0.94). After 4 weeks of sacubitril/valsartan treatment, the levels of urinary cyclic guanosine monophosphate and serum BNP were increased, whereas the levels of NT-proBNP and troponin T were decreased [24], suggesting that ARNI provide clinical benefits early during the course of treatment.

One of the limitations associated with the PARADIGM-HF trial was that the study population included participants who could tolerate the maximal dose of sacubitril/valsartan. However, many patients with $\mathrm{HFrEF}$ experience hypotension, which is a well-known predictor of morbidity and mortality in this population [25,26]. The TITRATION study was a double-blind RCT that assessed the safety and tolerability of ARNI in the real world, representative to routine practice, comparing two up-titration regimens [27]. A total of 498 patients with an LVEF $\leq 35 \%$ were enrolled and treated with sacubitril/ valsartan for 12 weeks. In that study, neither an elevated NP level nor prior use of ACEIs/ARBs was required for eligibility. As a result, $50.4 \%$ of participants had used low-dose ACEIs/ARBs, and 6.6\% were ACEI/ARB naïve. The condensed regimen consisted of $100 \mathrm{mg}$ of sacubitril/valsartan twice daily for 2 weeks followed by $200 \mathrm{mg}$ twice daily, while the conservative regimen started with $50 \mathrm{mg}$ twice daily for 2 weeks followed by $100 \mathrm{mg}$ twice daily for 3 weeks and then $200 \mathrm{mg}$ twice daily. Overall, 
75.9\% of patients completed the treatment regimen, reaching and maintaining the sacubitril/valsartan dose of $200 \mathrm{mg}$ twice per day over 12 weeks. Patients randomized to the condensed regimen had an increased frequency of adverse events such as hypotension, renal dysfunction, hyperkalemia and angioedema compared with the conservative regimen; however, the difference was not statistically significant.

Although patients who switched from the low-dose ACEI/ARB regimen to the condensed regimen had a significantly increased risk of hypotension (systolic blood pressure $[\mathrm{SBP}]<95 \mathrm{mmHg}$ ) and lower rates of achieving treatment success, the up-titration was successful in most patients regardless of the previous use or dose of ACEIs/ARBs [27]. The beneficial effects of sacubitril/ valsartan were consistent among all baseline SBP groups in the PARADIGM-HF trial [26], and patients with low SBP (100 to $110 \mathrm{mmHg}$ ) at screening did not influence the rate of treatment success in the TITRATION study [28]. These findings indicate that the use of ARNI in HF patients with low SBP should be considered based on the same criteria used for those with normal or high SBP; however, careful up-titration is necessary in patients with low SBP or taking low-dose ACEIs/ARBs.

\section{Acute heart failure}

The Comparison of Sacubitril-Valsartan versus Enalapril on Effect on NT-proBNP in Patients Stabilized from an Acute Heart Failure Episode (PIONEER-HF) trial was a double-blind, multi-center RCT that evaluated the efficacy and safety of sacubitril/valsartan treatment for 8 weeks in patients hospitalized for acute decompensated HF. A total of 881 patients with an LVEF $\leq 40 \%$ and NT-proBNP level $\geq 1,600 \mathrm{pg} / \mathrm{mL}$ or BNP level $\geq 400 \mathrm{pg} / \mathrm{mL}$ who were hospitalized for acute decompensated HF were randomly assigned to receive sacubitril/valsartan or enalapril treatment. The primary endpoint of the study was the time-averaged proportional change in the NT-proBNP level. The study found a greater reduction in the NT-proBNP concentration in the sacubitril/valsartan group compared with the enalapril group; the ratio of the NT-proBNP levels at 4 and 8 weeks (geometric mean) to the baseline level was 0.53 in the sacubitril/valsartan group and 0.75 in the enalapril group (ratio of change with sacubitril/valsartan vs. enalapril was 0.71 ; 95\% CI, 0.63 to 0.81 ; $p<0.001$ ). More- over, the change in the NT-proBNP level was evident within the first week of treatment. The sacubitril/valsartan group also had a greater reduction in the high-sensitivity troponin $\mathrm{T}$ level compared with the enalapril group (ratio of change, $0.85 ; 95 \% \mathrm{CI}, 0.77$ to 0.94 ). In an analysis of exploratory clinical outcomes, sacubitril/valsartan reduced the risk of rehospitalization for $\mathrm{HF}$ (HR, 0.56; 95\% CI, 0.37 to 0.84 ), but not death (HR, o.66; $95 \%$ CI, 0.30 to 1.48) (Table 2 and Fig. 2) [29].

A recent analysis of the PIONEER-HF trial evaluated the effect of sacubitril/valsartan compared with enalapril on adjudicated outcomes executed by the blinded clinical events committee. The outcomes including death, rehospitalization for HF, left ventricular assist device implantation, and listing for cardiac transplantation were less frequent in the sacubitril/valsartan group compared with the enalapril group (HR, 0.58; 95\% CI, 0.40 to $0.85 ; p=0.005)$. The rate of rehospitalization for $\mathrm{HF}$ and cardiovascular death was also significantly reduced in the sacubitril/valsartan group (HR, 0.58; 95\% CI, 0.39 to $0.87 ; p=0.007$ ) [30]. Since pharmacologic treatments including diuretics, vasodilators and inotropes did not improve the survival of acute HF patients [5], these results suggest that early initiation of ARNI for acute decompensated HF may reduce the risks of rehospitalization and cardiovascular death. However, cautious interpretation is needed because the primary endpoint of the PIONEER-HF trial was not related to these clinical outcomes.

Several studies support the early initiation of sacubitril/valsartan in patients hospitalized for acute decompensated HF. All participants in the PIONEER-HF trial completed 4 weeks of sacubitril/valsartan treatment following the 8-week study duration per protocol [31]. DeVore et al. [32] investigated this open-label extension treatment of sacubitril/valsartan and found similar levels of NT-proBNP between the enalapril and sacubitril/ valsartan groups after 4 weeks of ARNI treatment. However, the difference in the risk of cardiovascular death or rehospitalization for HF between the groups remained at 12 weeks following randomization (HR, 0.69; 95\% CI, 0.49 to $0.97 ; p=0.032$ ) [32]. In another sub-study of the PIONEER-HF trial, soluble ST2 and high-sensitivity cardiac troponin $\mathrm{T}$, which are considered surrogate markers of myocardial stress and injury, were significantly reduced in the sacubitril/valsartan group 
at the 1- and 4-week follow-up timepoints. Interestingly, the risk of cardiovascular death or rehospitalization for HF at week 8 was associated with the levels of these biomarkers at week 1 (high-sensitivity cardiac troponin T: HR, 1.34; 95\% CI, 1.001 to $1.81 ; p=0.049$ ) (soluble ST2: HR, 2.13; 95\% CI, 1.31 to $3.45 ; p=0.002$ ) (NT-proBNP: HR, $1.87 ; 95 \%$ CI, 1.46 to $2.40 ; p<0.001$ ) [33]. These findings provide further support for the early initiation of ARNI in patients hospitalized for acute HF.

The PIONEER-HF trial also demonstrated that the risks of adverse events, including worsening renal function, hyperkalemia, symptomatic hypotension and angioedema, were similar between the ARNI and enalapril treatment groups [29], suggesting it is safe to start ARNI during the index admission in acute HF patients after stabilization. The TRANSITION study evaluated the tolerability and safety of ARNI by initiating treatment at different timepoints in patients hospitalized for acute HF. In this trial, 1,002 patients with an LVEF $\leq 40 \%$ who were hospitalized for acute decompensated HF were randomized to initiate sacubitril/valsartan more than 12 hours before discharge versus within 14 days after discharge. In most patients, the starting dose of $50 \mathrm{mg}$ twice daily was used (88.4\% in the pre-discharge group and $84.5 \%$ in the post-discharge group). The percentage of patients who achieved the target dose of $200 \mathrm{mg}$ twice daily at the end of week 10 was similar between the groups (45.4\% in the pre-discharge group and 50.7\% in the post-discharge group; relative risk ratio [RRR], 0.90; $95 \% \mathrm{CI}, 0.79$ to 1.02; $p=0.099$ ). Moreover, the rates of maintaining any dose of ARNI for more than 2 weeks were $86.0 \%$ and $89.6 \%$ in the pre-discharge group and the post-discharge group, respectively (RRR, o.96; $95 \%$ CI, 0.92 to $1.01 ; p=0.089$ ), and the incidence of ARNI discontinuation due to adverse events was low in both groups $(7.3 \%$ for the pre-discharge group and $4.9 \%$ for the post-discharge group; RRR, 1.49; $95 \% \mathrm{CI}, 0.90$ to 2.46; $p=0.177$ ) (Table 2) [34].

In the TRANSITION study, $29 \%$ of patients were newly diagnosed with HFrEF. Senni et al. [35] investigated the differences in characteristics and prognosis between patients with de novo HFrEF and those with prior HFrEF in the TRANSITION population. The patients with de novo HFrEF were younger and had higher diastolic blood pressure, more preserved renal function, fewer comorbidities and milder symptoms compared with those with prior HF. The analysis showed that patients with de novo HFrEF were more likely to achieve the target dose of $200 \mathrm{mg}$ twice daily (RRR, 1.30; 95\% CI, 1.12 to $1.52 ; p<0.001$ ) and less likely to discontinue ARNI due to adverse events (RRR, 0.42; 95\% CI, 0.21 to $0.85 ; p=0.012$ ) compared with those with prior HFrEF. As expected, the incidences of cardiovascular death and rehospitalization for HF were significantly higher in the prior HFrEF group $(p=0.003)$; however, this finding may be attributed to the unfavorable baseline characteristics of the patients in the prior HFrEF group. Nevertheless, greater reductions in NT-proBNP and high-sensitivity troponin T levels at weeks 4 and 8 were seen in the de novo than in the prior HFrEF groups, which may also have contributed to the improved clinical outcomes of the de novo HFrEF population [35].

\section{Heart failure with preserved ejection fraction}

The Prospective comparison of ARNI with ARB on the Management Of heart failUre with preserved ejectioN fracTion (PARAMOUNT) trial was a phase 2 double-blind RCT assessing the efficacy and safety of sacubitril/valsartan compared with valsartan among patients with $\mathrm{HF}$ with preserved ejection fraction (HFpEF). The trial enrolled 301 patients with NYHA class II-III, $\mathrm{LVEF} \geq 45 \%$ and NT-proBNP level $>400 \mathrm{pg} / \mathrm{mL}$. The sacubitril/valsartan group showed a significantly greater reduction in the NT-proBNP level compared with the valsartan group (ratio of change of NT-proBNP at 12 weeks for ARNI vs. valsartan, 0.77 ; 95\% CI, 0.64 to 0.92 ; $p=0.005)$. In addition, the reductions in the left atrial volume and dimensions were greater in the sacubitril/ valsartan group compared with the valsartan group after 36 weeks of treatment, and adverse events were similar between the groups [36].

Similar to the PARAMOUNT trial, the Prospective Comparison of ARNI with ARB Global Outcomes in HF With Preserved Ejection Fraction (PARAGON-HF) trial evaluated the efficacy and safety of ARNI in HFpEF patients. Unlike previous trials of ARNI both studies used valsartan as the active comparator [37]. In the PARAGON-HF trial, a total of 4,822 HF patients with NYHA class II-III, LVEF $\geq 45 \%$ and elevated NT-proBNP level were enrolled. The primary composite endpoints were total hospitalization for HF and cardiovascular death. The incidence of the primary endpoint was lower but 
not statistically significant in the sacubitril/valsartan group, with a rate ratio of 0.87 (95\% CI, 0.75 to 1.01; $p=$ o.06). Moreover, ARNI treatment did not decrease the risk of all-cause (HR, 0.97; 95\% CI, 0.84 to 1.13) or cardiovascular (HR, 0.95; 95\% CI, 0.79 to 1.16) death in HFpEF patients (Table 1 and Fig. 2) [38].

As none of the pharmacological therapies showed a survival benefit in patients with HFpEF, the results of the PARAGON-HF were discouraging for clinicians who expected optimistic results following the PARADIGM-HF trial. One of the possible explanations for the negative findings in the PARAGON-HF trial was the heterogeneity in the HFpEF population and the complex pathophysiology of HFpEF [39,40]. These findings may also be explained by the lower levels of NT-proBNP in patients in the PARAGON-HF trial compared with the PARADIGM-HF trial [41], as previous reports suggest that a lower NT-proBNP level may arise from reduced wall stress in HFpEF compared with HFrEF [42]. Moreover, assuming that the beneficial effect of ARNI in HFrEF patients is associated with reduced adverse remodeling of the left ventricle following NP system activation, this mechanism may be attenuated in HFpEF patients, who often have smaller left ventricles and lower NP levels [41]. A recent study demonstrated that the level of soluble neprilysin is lower in patients with HFpEF than in healthy controls [43], which may also explain the reduced effects of neprilysin inhibition among HFpEF patients. Despite these findings, the PARAGON-HF trial showed a small reduction in the incidence of total hospitalization for HF in the ARNI treatment group, suggesting potential benefits in subpopulations of HFpEF patients. Further large-scale clinical trials with refined inclusion criteria are needed.

In the prespecified subgroup analysis, female patients and patients with LVEF $\leq 57 \%$ showed greater reduction in the primary endpoint after ARNI treatment, suggesting that subsets of HFpEF patients may benefit from sacubitril/valsartan [38]. In the post hoc analysis, ARNI reduced the risk of hospitalization for HF in women (HR, 0.73; 95\% CI, 0.59 to 0.90) but not in men (HR, 1.03; 95\% CI, 0.84 to 1.25) with HFpEF [44]. Recently, the results from an individual patient-level pooled analysis of the PARADIGM-HF and PARAGON-HF trials were published. The analyses found that the LVEF significantly affected the composite outcome in the ARNI treatment group (interaction $p$ for treatment-by-continuous LVEF = 0.02), and sacubitril/valsartan was beneficial in patients with an LVEF below the normal range [45]. Moreover, the echocardiographic features of patients in the PARAGON-HF trial were different from those in previous trials of HFpEF, highlighting the heterogeneity within the HFpEF population [40]. Therefore, more detailed analyses and follow-up studies are necessary to find the patients who are beneficial from ARNI treatment among HFpEF patients. New HF classification methods beyond the LVEF may be required to identify the appropriate subgroups who benefit from contemporary HF treatments.

\section{THE ADDITIONAL EFFECTS OF ANGIOTENSIN RECEPTOR-NEPRILYSIN INHIBITOR}

\section{Reverse remodeling of the left ventricle}

A possible mechanism of the beneficial effects conferred by ARNI treatment is left ventricular remodeling. Increased LVEF and decreased left ventricular volumes induced by drugs or devices used for HF are associated with long-term mortality in patients with an LVEF $\leq 45 \%$ [46]. A recent meta-analysis demonstrated the effect of ARNI on reverse cardiac remodeling. In that study, HFrEF patients treated with ARNI showed improvements in the LVEF and most cardiac remodeling indices, including left ventricular end-diastolic volume, left ventricular end-systolic volume, left atrial volume, and left ventricular mass index, compared with patients treated with ACEIs or ARBs [47].

Moreover, the PARADIGM-HF trial demonstrated a rapid decrease in the NT-proBNP level and reduced mortality after ARNI treatment, which may also be associated with left ventricular remodeling. The Prospective Study of Biomarkers, Symptom Improvement, and Ventricular Remodeling During Sacubitril/Valsartan Therapy for Heart Failure (PROVE-HF) was a single-arm study that assessed the correlation between NT-proBNP level changes and cardiac remodeling in 794 patients with HFrEF treated with ARNI. The study found a significant increase in the LVEF and decreases in left ventricular and left atrial volumes after 6 and 12 months of treatment. The reduction in NT-proBNP was also associated with reverse remodeling of the 
myocardium ( $r=0.430$ for NT-proBNP vs. left ventricular diastolic volume index) [48].

\section{Functional mitral regurgitation}

The Pharmacological Reduction of Functional, Ischemic Mitral REgurgitation (PRIME) study was a double-blind RCT that compared sacubitril/valsartan with valsartan treatment in patients with $\mathrm{HF}$ and chronic functional mitral regurgitation. A total of 118 patients with an LVEF of $25 \%$ to $50 \%$ and severe chronic functional mitral regurgitation were treated with the study drugs for 12 months. The effective regurgitant orifice area and regurgitant volume were significantly decreased after treatment in the sacubitril/valsartan group compared with the valsartan group (effective regurgitant orifice area: $-0.058 \pm 0.095 \mathrm{~cm}^{2}$ vs. $-0.018 \pm 0.105 \mathrm{~cm}^{2}, p=0.032$; regurgitant volume: $-11.6 \pm 14.4 \mathrm{~mL}$ vs. $-4.3 \pm 15.1 \mathrm{~mL}, p$ $=0.009)$. In addition, the sacubitril/valsartan group had a decreased left ventricular end-diastolic volume index ( $p=0.044)$ [49]. The study showed that small changes in left ventricular volume induced by ARNI may be beneficial in reducing the functional mitral regurgitation. Further research is needed to evaluate the effect of ARNI treatment on patient-centered outcomes associated with functional mitral regurgitation.

\section{Aortic stiffness}

Another potential mechanism underlying the beneficial effect of ARNI treatment is a reduction in aortic stiffness. In older hypertensive patients, sacubitril/valsartan significantly reduced aortic stiffness compared with olmesartan after 12 weeks of treatment [50]. Based on these findings, the EVALUATE-HF trial aimed to evaluate the effects of sacubitril/valsartan compared with enalapril on aortic stiffness in HFrEF patients. However, sacubitril/valsartan did not significantly reduce aortic characteristic impedance compared with enalapril. The authors suggested that these findings may be attributed to differences between HFrEF and hypertensive patients, as the former may have lower levels of aortic stiffness due to the activated NP system [51].

\section{Protection of renal function and increased albuminuria} In a post hoc analysis of the PARADIGM-HF trial, Damman et al. [52] showed that sacubitril/valsartan resulted in improved outcomes among patients with chronic kidney disease (CKD). The estimated glomerular filtration rate (eGFR) decreased by $10.2 \mathrm{~mL} / \mathrm{min} / 1.73 \mathrm{~m}^{2}$ (95\% CI, 12.1 to 8.3) in patients assigned to enalapril between screening and end of follow-up and by $7.8 \mathrm{~mL} / \mathrm{min} / 1.73 \mathrm{~m}^{2}(95 \%$ CI, 9.6 to 6.0) in those assigned to sacubitril/valsartan. However, the urinary albumin/creatinine ratio (uACR) was significantly increased during the run-in period and remained elevated in patients treated with sacubitril/ valsartan, whereas the uACR was decreased in patients treated with enalapril after randomization. Moreover, patients with a worsened uACR experienced higher rates of pre-specified renal outcomes in the enalapril group compared with the sacubitril/valsartan group [52]. HFpEF patients in the sacubitril/valsartan group had similar results, with a smaller reduction in the eGFR and a greater increase in the uACR compared with the valsartan group after 36 weeks of treatment [53]. This result was consistent between those with and those without diabetes. Packer et al. [54] showed that sacubitril/valsartan was associated with a lesser decrement of eGFR than enalapril, and the decrease in eGFR was smaller in patients without diabetes than the patients with diabetes.

Although both the dilation of efferent arterioles and the hypotensive effect by agents that block the renin-angiotensin system can result in a reduction in the eGFR, ARNI treatment mitigated this effect. This may be attributed to activated NP system by ARNI, which affects the complex glomerular hemodynamics [53,55]. Moreover, a similar mechanism may explain the increased uACR in the ARNI treatment group. Although an increased uACR is often considered a marker of glomerular damage in CKD patients, the increased uACR during the run-in period returned to the screening level after 1 month of enalapril treatment, which suggests that these changes are reversible. Furthermore, the increased uACR did not decrease the eGFR and did not affect the clinical outcomes [52]. Therefore, the prognostic significance of an increased UACR in HF patients treated with ARNI remains unclear, and extrapolating the renal effect of ARNI beyond HF requires caution. In patients with CKD, both sacubitril/valsartan and irbesartan resulted in decreased eGFR and uACR [56].

\section{Glycemic control}

In another post hoc analysis of the PARADIGM-HF trial, ARNI treatment resulted in improved glycemic control 
in HFrEF patients with known diabetes or an hemoglobin A1c (HbA1c) level $\geq 6.5 \%$ [57]. The reduction in the $\mathrm{HbAlc} \mathrm{level} \mathrm{was} \mathrm{greater} \mathrm{in} \mathrm{the} \mathrm{sacubitril/valsartan} \mathrm{group}$ than in the enalapril group after 3 years of treatment ( $7.41 \%$ at baseline to $6.97 \%$ at 3 years vs. $7.48 \%$ at baseline to $7.16 \%$ at 3 years). Moreover, the proportion of patients who initiated insulin therapy was significantly lower in the sacubitril/valsartan group compared with the enalapril group (HR, 0.71; 95\% CI, 0.56 to $0.90 ; p=0.0052)$ [57]. Although the PARADIGM-HF trial was not designed to evaluate the efficacy of glycemic control, the small reduction in the HbAlc level is meaningful, as the management of diabetes was left to the discretion of the physician. The mechanism of glycemic control may be linked to various substrates of neprilysin, including angiotensin I and II, glucagon-like peptide 1 (GLP-1) and bradykinin, which can improve insulin sensitivity and cause vasodilation via various pathways.

\section{ANGIOTENSIN RECEPTOR-NEPRILYSIN INHIBITOR IN RECENT UPDATES OF HEART FAILURE GUIDELINES}

\section{Chronic symptomatic heart failure}

Growing evidence regarding ARNI treatment has led to several changes in HF treatment guidelines. ARNI first appeared in the 2016 European Society of Cardiology (ESC) guidelines for HF, which referenced the results of the PARADIGM-HF trial published in 2014. ARNI is recommended to replace ACEI in ambulatory, symptomatic patients with HFrEF despite optimal therapy with ACEI, beta-blocker, and mineralocorticoid receptor antagonist in ESC guideline (class I, level of evidence B) [5]. The 2017 focused update of the 2013 American College of Cardiology/American Heart Association guidelines included the same recommendation (class I, level of evidence B-R) [6]. The Korean Society of Heart Failure updated the guideline for chronic HF in 2018, which recommended replacing ACEIs or ARBs with ARNI in patients with chronic HFrEF NYHA functional class II or III to reduce mortality (class I, level of evidence B-R) [58].

\section{Acute heart failure}

The aforementioned guidelines made no recommen- dation regarding ARNI for patients hospitalized with decompensated HF. Following publication of the PIONEER-HF trial in 2019, the expert consensus document from ESC [59] and expert consensus decision pathway from the American College of Cardiology [6o] stated that the initiation of sacubitril/valsartan instead of ACEIs or ARBs may be considered for patients admitted for decompensated HF. Although the Korean Food and Drug Administration label has not included the use of sacubitril/valsartan in acute HF patients to date, the Korean Society of Heart Failure recently revised the guideline to consider sacubitril/valsartan as an initial treatment for patients hospitalized with acute decompensated HF (class IIb, level of evidence B). However, the main barrier to ARNI use for acute decompensated HF in Korea is the limited adherence to guideline-directed medical therapies [61].

\section{ONGOING RANDOMIZED CONTROLLED TRIALS OF ANGIOTENSIN RECEPTOR- NEPRILYSIN INHIBITOR FOR HEART FAILURE}

The Entresto ${ }^{\text {TM }}$ (LCZ696) in Advanced Heart Failure (LIFE Study) (HFN-LIFE) trial (NCTo2816736) is enrolling patients with advanced HFrEF NYHA class IV symptoms and high NT-proBNP levels. The primary endpoint is the change in the NT-proBNP level after 24 weeks of treatment with sacubitril/valsartan or valsartan. Since only $0.7 \%$ of patients enrolled in the PARADIGM-HF trial were NYHA class IV [4], the HFNLIFE trial will provide new evidence on the efficacy and safety of ARNI in patients with advanced HF.

The Prospective ARNI vs. ACE Inhibitor Trial to DetermIne Superiority in Reducing Heart Failure Events After MI (PARADISE-MI) (NCTo2924727) is evaluating sacubitril/valsartan and ramipril in patients with an LVEF $\leq 40 \%$ and/or pulmonary congestion after acute myocardial infarction. The primary composite endpoint is the occurrence of cardiovascular death, hospitalization for HF or outpatient HF.

The Changes in NT-proBNP and Outcomes, Safety, and Tolerability in HFpEF Patients With Acute Decompensated Heart Failure (ADHF) Who Have Been Stabilized During Hospitalization and Initiated In-hospital or Within 30 Days Post-discharge (PARAGLIDE-HF) trial 


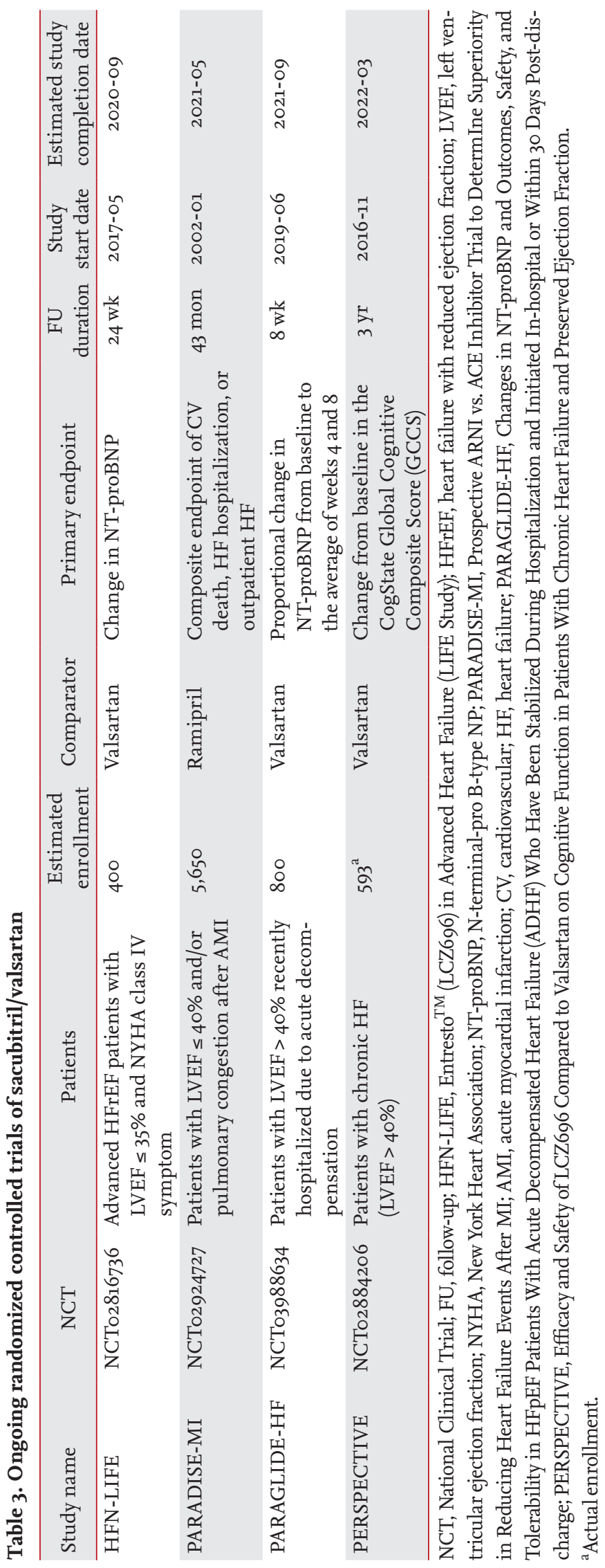

(NCTo3988634) is a double-blind RCT comparing the efficacies of sacubitril/valsartan and valsartan in HFpEF patients hospitalized for acute decompensated HF. This trial closely resembles the PIONEER-HF trial but only enrolled patients with an LVEF > 40\% within the past 3 months. The primary endpoint is the change in the NT-proBNP level after 4 and 8 weeks of treatment.

The Efficacy and Safety of LCZ696 Compared to Valsartan on Cognitive Function in Patients With Chronic Heart Failure and Preserved Ejection Fraction (PERSPECTIVE) trial (NCTo2884206) is evaluating the efficacy and safety of sacubitril/valsartan in terms of cognitive function compared with valsartan in patients with HFpEF after 3 years of treatment. Since neprilysin is involved in the degradation of amyloid- $\beta$ peptides, the inhibition of neprilysin may be associated with cognitive decline. However, the post hoc analysis of the PARADIGM-HF trial showed that the development of cognitive dysfunction was similar between the sacubitril/valsartan and enalapril groups [62]. Nevertheless, further longitudinal studies assessing the long-term effects of ARNI are warranted. Results of the PERSPECTIVE trial and post hoc analysis of the PARAGON-HF trial will further our understanding of the effects of ARNI on cognitive function in patients with $\mathrm{HFpEF}$ (Table 3).

\section{CONCLUSIONS}

Sacubitril/valsartan treatment compensates for the limitations of existing HF treatments, as this regimen targets the third axis of the neurohormonal control in HF. ARNI has shifted the HF treatment paradigm via large-scale RCTs and has demonstrated improvements over existing therapies such as renin-angiotensin system blockers. Post hoc analyses of the PARADIGM-HF trial and subsequent studies have helped elucidate the mechanism underlying the beneficial effects of sacubitril/valsartan treatment. Moreover, the indications for ARNI are expanding, as a result of studies conducted in patients with the full spectrum of HF. Ongoing trials will help address several remaining questions. Longterm follow-up studies are needed to investigate the sustainability of benefits and delayed adverse effects of ARNI in patient with HF. 


\section{Conflict of interest}

No potential conflict of interest relevant to this article was reported.

\section{REFERENCES}

1. Lee JH, Lim NK, Cho MC, Park HY. Epidemiology of heart failure in Korea: present and future. Korean Circ J 2016;46:658-664.

2. Choi HM, Park MS, Youn JC. Update on heart failure management and future directions. Korean J Intern Med 2019;34:11-43.

3. Levy D, Kenchaiah S, Larson MG, et al. Long-term trends in the incidence of and survival with heart failure. N Engl J Med 2002;347:1397-1402.

4. McMurray JJ, Packer M, Desai AS, et al. Angiotensin-neprilysin inhibition versus enalapril in heart failure. N Engl J Med 2014;371:993-1004.

5. Ponikowski P, Voors AA, Anker SD, et al. 2016 ESC guidelines for the diagnosis and treatment of acute and chronic heart failure: the task force for the diagnosis and treatment of acute and chronic heart failure of the European Society of Cardiology (ESC) Developed with the special contribution of the Heart Failure Association (HFA) of the ESC. Eur Heart J 2016;37:2129-2200.

6. Yancy CW, Jessup M, Bozkurt B, et al. 2017 ACC/AHA/ HFSA focused update of the 2013 ACCF/AHA guideline for the management of heart failure: a report of the American College of Cardiology/American Heart Association task force on clinical practice guidelines and the heart failure society of America. Circulation 2017;136:e137-e161.

7. Kim JJ, Youn JC. Eligibility and usage of sacubitril/valsartan in Korea. Int J Heart Fail 2019;1:69-71.

8. Mann DL, Bristow MR. Mechanisms and models in heart failure: the biomechanical model and beyond. Circulation 2005;111:2837-2849.

9. Hartupee J, Mann DL. Neurohormonal activation in heart failure with reduced ejection fraction. Nat Rev Cardiol 2017;14:30-38.

10. Packer M. The neurohormonal hypothesis: a theory to explain the mechanism of disease progression in heart failure. J Am Coll Cardiol 1992;20:248-254.

11. Campbell DJ. Long-term neprilysin inhibition: implications for ARNIs. Nat Rev Cardiol 2017;14:171-186.
12. O'Connor CM, Starling RC, Hernandez AF, et al. Effect of nesiritide in patients with acute decompensated heart failure. N Engl J Med 2011;365:32-43.

13. Bayes-Genis A, Lupon J. Neprilysin: indications, expectations, and challenges. Rev Esp Cardiol (Engl Ed) 2016;69:647-649.

14. Cleland JG, Swedberg K. Lack of efficacy of neutral endopeptidase inhibitor ecadotril in heart failure. The International Ecadotril Multi-centre Dose- ranging Study Investigators. Lancet 1998;351:1657-1658.

15. Greenberg B. Angiotensin receptor-neprilysin inhibition (ARNI) in heart failure. Int J Heart Fail 2020;2:73-90.

16. Packer M, Califf RM, Konstam MA, et al. Comparison of omapatrilat and enalapril in patients with chronic heart failure: the omapatrilat versus enalapril randomized trial of utility in reducing events (OVERTURE). Circulation 2002;106:920-926.

17. Kostis JB, Packer M, Black HR, Schmieder R, Henry D, Levy E. Omapatrilat and enalapril in patients with hypertension: the omapatrilat cardiovascular treatment vs. enalapril (OCTAVE) trial. Am J Hypertens 2004;17:103-111.

18. Nussberger J, Cugno M, Amstutz C, Cicardi M, Pellacani A, Agostoni A. Plasma bradykinin in angio-oedema. Lancet 1998;351:1693-1697.

19. McMurray JJ, Packer M, Desai AS, et al. Dual angiotensin receptor and neprilysin inhibition as an alternative to angiotensin-converting enzyme inhibition in patients with chronic systolic heart failure: rationale for and design of the Prospective comparison of ARNI with ACEI to Determine Impact on Global Mortality and morbidity in Heart Failure trial (PARADIGM-HF). Eur J Heart Fail 2013;15:1062-1073.

20. SOLVD Investigators, Yusuf S, Pitt B, Davis CE, Hood WB, Cohn JN. Effect of enalapril on survival in patients with reduced left ventricular ejection fractions and congestive heart failure. N Engl J Med 1991;325:293-302.

21. Desai AS, McMurray JJ, Packer M, et al. Effect of the angiotensin-receptor-neprilysin inhibitor LCZ696 compared with enalapril on mode of death in heart failure patients. Eur Heart J 2015;36:1990-1997.

22. Sarrias A, Bayes-Genis A. Is sacubitril/valsartan (also) an antiarrhythmic drug? Circulation 2018;138:551-553.

23. Chang PC, Lin SF, Chu Y, et al. LCZ696 therapy reduces ventricular tachyarrhythmia inducibility in a myocardial infarction-induced heart failure rat model. Cardiovasc Ther 2019;2019:6032631. 
24. Packer M, McMurray JJ, Desai AS, et al. Angiotensin receptor neprilysin inhibition compared with enalapril on the risk of clinical progression in surviving patients with heart failure. Circulation 2015;131:54-61.

25. Gheorghiade M, Abraham WT, Albert NM, et al. Systolic blood pressure at admission, clinical characteristics, and outcomes in patients hospitalized with acute heart failure. JAMA 2006;296:2217-2226.

26. Bohm M, Young R, Jhund PS, et al. Systolic blood pressure, cardiovascular outcomes and efficacy and safety of sacubitril/valsartan (LCZ696) in patients with chronic heart failure and reduced ejection fraction: results from PARADIGM-HF. Eur Heart J 2017;38:1132-1143.

27. Senni M, McMurray JJ, Wachter R, et al. Initiating sacubitril/valsartan (LCZ696) in heart failure: results of TITRATION, a double-blind, randomized comparison of two uptitration regimens. Eur J Heart Fail 2016;18:1193-1202.

28. Senni M, McMurray JJV, Wachter R, et al. Impact of systolic blood pressure on the safety and tolerability of initiating and up-titrating sacubitril/valsartan in patients with heart failure and reduced ejection fraction: insights from the TITRATION study. Eur J Heart Fail 2018;20:491500.

29. Velazquez EJ, Morrow DA, DeVore AD, et al. Angiotensin-neprilysin inhibition in acute decompensated heart failure. N Engl J Med 2019;380:539-548.

30. Morrow DA, Velazquez EJ, DeVore AD, et al. Clinical outcomes in patients with acute decompensated heart failure randomly assigned to sacubitril/valsartan or enalapril in the PIONEER-HF trial. Circulation 2019;139:2285-2288.

31. Velazquez EJ, Morrow DA, DeVore AD, et al. Rationale and design of the comParIson Of sacubitril/valsartaN versus Enalapril on Effect on nt-pRo-bnp in patients stabilized from an acute Heart Failure episode (PIONEER-HF) trial. Am Heart J 2018;198:145-151.

32. DeVore AD, Braunwald E, Morrow DA, et al. Initiation of angiotensin-neprilysin inhibition after acute decompensated heart failure: secondary analysis of the open-label extension of the PIONEER-HF trial. JAMA Cardiol 2019;5:202-207.

33. Morrow DA, Velazquez EJ, DeVore AD, et al. Cardiovascular biomarkers in patients with acute decompensated heart failure randomized to sacubitril-valsartan or enalapril in the PIONEER-HF trial. Eur Heart J 2019;40:33453352.

34. Wachter R, Senni M, Belohlavek J, et al. Initiation of sacubitril/valsartan in haemodynamically stabilized heart failure patients in hospital or early after discharge: primary results of the randomised TRANSITION study. Eur J Heart Fail 2019;21:998-1007.

35. Senni M, Wachter R, Witte KK, et al. Initiation of sacubitril/valsartan shortly after hospitalisation for acutely decompensated heart failure in patients with newly diagnosed (de novo) heart failure: a subgroup analysis of the TRANSITION study. Eur J Heart Fail 2020;22:303-312.

36. Solomon SD, Zile M, Pieske B, et al. The angiotensin receptor neprilysin inhibitor LCZ696 in heart failure with preserved ejection fraction: a phase 2 double-blind randomised controlled trial. Lancet 2012;380:1387-1395.

37. Solomon SD, Rizkala AR, Gong J, et al. Angiotensin receptor neprilysin inhibition in heart failure with preserved ejection fraction: rationale and design of the PARAGON-HF trial. JACC Heart Fail 2017;5:471-482.

38. Solomon SD, McMurray JJV, Anand IS, et al. Angiotensin-neprilysin inhibition in heart failure with preserved ejection fraction. N Engl J Med 2019;381:1609-1620.

39. Shah SJ, Katz DH, Selvaraj S, et al. Phenomapping for novel classification of heart failure with preserved ejection fraction. Circulation 2015;131:269-279.

40. Shah AM, Cikes M, Prasad N, et al. Echocardiographic features of patients with heart failure and preserved left ventricular ejection fraction. J Am Coll Cardiol 2019;74:28582873 .

41. O'Connor CM, deFilippi C. PARAGON-HF: why we do randomized, controlled clinical trials. N Engl J Med 2019;381:1675-1676.

42. Kang SH, Park JJ, Choi DJ, et al. Prognostic value of NT-proBNP in heart failure with preserved versus reduced EF. Heart 2015;101:1881-1888.

43. Lyle MA, Iyer SR, Redfield MM, et al. Circulating neprilysin in patients with heart failure and preserved ejection fraction. JACC Heart Fail 2020;8:70-80.

44. McMurray JJV, Jackson AM, Lam CSP, et al. Effects of sacubitril-valsartan versus valsartan in women compared with men with heart failure and preserved ejection fraction: insights from PARAGON-HF. Circulation 2020;141:338-351.

45. Solomon SD, Vaduganathan M, Claggett BL, et al. Sacubitril/valsartan across the spectrum of ejection fraction in heart failure. Circulation 2020;141:352-361.

46. Kramer DG, Trikalinos TA, Kent DM, Antonopoulos GV, Konstam MA, Udelson JE. Quantitative evaluation 
of drug or device effects on ventricular remodeling as predictors of therapeutic effects on mortality in patients with heart failure and reduced ejection fraction: a meta-analytic approach. J Am Coll Cardiol 2010;56:392-406.

47. Wang Y, Zhou R, Lu C, Chen Q, Xu T, Li D. Effects of the angiotensin-receptor neprilysin inhibitor on cardiac reverse remodeling: meta-analysis. J Am Heart Assoc 2019;8:e012272.

48. Januzzi JL Jr, Prescott MF, Butler J, et al. Association of change in n-terminal pro-b-type natriuretic peptide following initiation of sacubitril-valsartan treatment with cardiac structure and function in patients with heart failure with reduced ejection fraction. JAMA 2019;322:1-11.

49. Kang DH, Park SJ, Shin SH, et al. Angiotensin receptor neprilysin inhibitor for functional mitral regurgitation. Circulation 2019;139:1354-1365.

50. Williams B, Cockcroft JR, Kario K, et al. Effects of sacubitril/valsartan versus olmesartan on central hemodynamics in the elderly with systolic hypertension: the PARAMETER study. Hypertension 2017;69:411-420.

51. Desai AS, Solomon SD, Shah AM, et al. Effect of sacubitril-valsartan vs enalapril on aortic stiffness in patients with heart failure and reduced ejection fraction: a randomized clinical trial. JAMA 2019;322:1-10.

52. Damman K, Gori M, Claggett B, et al. Renal effects and associated outcomes during angiotensin-neprilysin inhibition in heart failure. JACC Heart Fail 2018;6:489-498.

53. Voors AA, Gori M, Liu LC, et al. Renal effects of the angiotensin receptor neprilysin inhibitor LCZ696 in patients with heart failure and preserved ejection fraction. Eur J Heart Fail 2015;17:510-517.

54. Packer M, Claggett B, Lefkowitz MP, et al. Effect of neprilysin inhibition on renal function in patients with type 2 diabetes and chronic heart failure who are receiving target doses of inhibitors of the renin-angiotensin system: a secondary analysis of the PARADIGM-HF trial. Lancet
Diabetes Endocrinol 2018;6:547-554.

55. Mullens W, Martens P. Exploiting the natriuretic peptide pathway to preserve glomerular filtration in heart failure. JACC Heart Fail 2018;6:499-502.

56. Haynes R, Judge PK, Staplin N, et al. Effects of sacubitril/ valsartan versus irbesartan in patients with chronic kidney disease. Circulation 2018;138:1505-1514.

57. Seferovic JP, Claggett B, Seidelmann SB, et al. Effect of sacubitril/valsartan versus enalapril on glycaemic control in patients with heart failure and diabetes: a post-hoc analysis from the PARADIGM-HF trial. Lancet Diabetes Endocrinol 2017;5:333-340.

58. Kim KJ, Cho HJ, Kim MS, et al. Focused update of 2016 Korean Society of Heart Failure guidelines for the management of chronic heart failure. Int J Heart Fail 2019;1:424.

59. Seferovic PM, Ponikowski P, Anker SD, et al. Clinical practice update on heart failure 2019: pharmacotherapy, procedures, devices and patient management: an expert consensus meeting report of the Heart Failure Association of the European Society of Cardiology. Eur J Heart Fail 2019;21:1169-1186.

6o. Hollenberg SM, Warner Stevenson L, Ahmad T, et al. 2019 ACC expert consensus decision pathway on risk assessment, management, and clinical trajectory of patients hospitalized with heart failure: a report of the American college of Cardiology solution set oversight committee. J Am Coll Cardiol 2019;74:1966-2011.

61. Oh J, Lee CJ, Park JJ, et al. Real-world eligibility for sacubitril/valsartan in heart failure with reduced ejection fraction patients in Korea: data from the Korean Acute Heart Failure (KorAHF) Registry. Int J Heart Fail 2019;1:57-68.

62. Cannon JA, Shen L, Jhund PS, et al. Dementia-related adverse events in PARADIGM-HF and other trials in heart failure with reduced ejection fraction. Eur J Heart Fail 2017;19:129-137. 